\title{
Sémantique selon la théorie des jeux et situations de validation en mathématiques
}

Thomas Barrier

\section{(2) OpenEdition \\ 12 Journals}

\section{Édition électronique}

URL : https://journals.openedition.org/educationdidactique/350

DOI : 10.4000/educationdidactique.350

ISBN : 978-2-7535-1619-9

ISSN : 2111-4838

\section{Éditeur}

Presses universitaires de Rennes

\section{Édition imprimée}

Date de publication : 1 décembre 2008

Pagination : $35-58$

ISBN : 978-2-7535-0790-6

ISSN : 1956-3485

\section{Référence électronique}

Thomas Barrier, « Sémantique selon la théorie des jeux et situations de validation en

mathématiques », Éducation et didactique [En ligne], 2-3 | Décembre 2008, mis en ligne le 01 décembre 2010, consulté le 16 août 2022. URL : http://journals.openedition.org/educationdidactique/350 ; DOI : https://doi.org/10.4000/educationdidactique.350 


\title{
SÉMANTIQUE SELON LA THÉORIE DES JEUX ET SITUATIONS DE VALIDATION EN MATHÉMATIQUES
}

\author{
Thomas Barrier, Université de Lyon, LEPS-LIRDHIST
}

\begin{abstract}
Résumé : Lobjectif de ce texte est de mettre en avant le potentiel de la sémantique selon la théorie des jeux (GTS) pour l'analyse didactique des dialogues d'élèves en situation de validation. Nous nous efforcerons de montrer l'adéquation de cette théorie, due au logicien et philosophe Hintikka, avec l'approche de la théorie des situations, en particulier avec le fait d'utiliser l'évolution des stratégies des élèves dans une situation donnée comme critère d'acquisition d'une connaissance. Notre hypothèse est que la sémantique GTS peut contribuer à fournir des modélisations susceptibles d'éclairer ces évolutions stratégiques.
\end{abstract}

Mots clés : sémantique GTS, logique dialogique, situation de validation, stratégie, didactique des mathématiques

\section{Introduction}

L'objectif de ce texte est de proposer et d'évaluer un outil pour l'analyse des dialogues d'élèves dans les moments de recherche en mathématiques et en particulier dans ce que Brousseau a appelé les "situations de validation explicite " (Brousseau, 1998, p. 109-112). Nous discuterons de l'intérêt de l'usage de la sémantique selon la théorie des jeux (sémantique GTS $^{1}$ ) de Hintikka pour mener l'analyse de cette classe de dialogues. Le besoin de ce type d'outils trouve sa source dans le positionnement épistémologique de la Théorie des Situations Didactiques Mathématiques ${ }^{2}$ qui considère l'évolution de la stratégie d'un élève dans une situation donnée comme le critère servant à l'évaluation d'un apprentissage. Pour évaluer les évolutions stratégiques des élèves, les traces écrites d'un travail de recherche sont insuffisantes ce qui conduit de nombreux chercheurs à utiliser des enregistrements audio, voir vidéo. Dans notre travail, nous considérons, avec DurandGuerrier, Hérault et Tisseron (2006) et Guernier, Durand-Guerrier et Sautot (2007), qu'une analyse fine des « jeux de langage »-selon le vocabulaire de Wittgenstein (2004) - est nécessaire. Nous faisons l'hypothèse que la sémantique GTS offre une référence épistémologique pertinente pour faire fonctionner le critère selon lequel les acquisitions de connaissances s'identifient avec des évolutions stratégiques dans une situation. Autrement dit, nous essayerons de montrer en quoi la sémantique selon la théorie des jeux permet une analyse de la dimension stratégique des dialogues.

Après avoir explicité la question, issue du positionnement épistémologique de la Théorie des situations, que cet article aborde ( $\S 1$ ), nous présenterons la logique dialogique de Lorenzen (1967) qui fait partie des références théoriques de Brousseau. Nous montrerons les possibilités de celle-ci ( $\$ 2$ ) mais également ses limites ( $\$ 3$ ) puisque, selon nous, cette approche n'est que partiellement satisfaisante. Notre ambition sera ensuite de proposer une réponse alternative, en appui sur la sémantique GTS et la correction de cette dernière par Vernant ${ }^{3}$, qui puisse être fonctionnelle là où la logique dialogique de Lorenzen ne l'est pas (§ 3). Nous terminerons cet article par une analyse du rôle conféré au point de vue sémantique dans la théorie des situations, en particulier dans les situations de validation (§ 4).

\section{Un questionnement issu de l'approche épistémologique de la théorie des situations}

Sur le plan épistémologique, Brousseau revendique une relation étroite entre connaissance et 
stratégie. Il dit par exemple dans son cours donné lors de l'attribution du titre de Docteur Honoris Causa de l'Université de Montréal en 1997 que « une connaissance pertinente modifie une tactique ou une stratégie » (Brousseau, 1997, p. 4). Cette approche, parente de celle dite anthropologique de Wittgenstein ${ }^{4}$ est un positionnement critique vis-àvis d'un usage incontrôlé du vocabulaire de la psychologie. Pour ces deux auteurs, ce sont les actions et notamment les assertions des individus qui sont révélatrices et constitutives de leurs connaissances. Ils partagent également le point de vue selon lequel les objets mathématiques et les discours sur ceux-ci prennent leur sens lorsqu'ils sont plongés dans des situations ${ }^{5}$. Une des ambitions de la théorie des situations est de modéliser les activités des élèves afin de permettre la description et l'évaluation des stratégies mises en œuvre dans la situation ou, dit autrement, de mesurer la conformité de l'usage par les élèves des termes avec les règles ${ }^{6}$ en usage dans l'institution. Au sujet de cette modélisation, Brousseau affirme dans le même cours:

«En conjuguant l'approche d'Alan Turing et celle de Paul Lorenzen qui semblaient pourtant irréductiblement opposées il a paru possible de représenter les situations à usage didactique par des « automates mathématiques ».» (p. 4)

Il donne ensuite l'exemple du jeu de la course à 20 et la modélisation qui lui est associée: le jeu est représenté par une succession d'états (un état $(\theta, \mathrm{n})$ est la donnée d'un joueur et d'un entier naturel plus petit que 20). À partir de là, il devient possible de repérer et d'évaluer les modifications stables de stratégie et par là de parvenir à une certaine évaluation des apprentissages. Cependant, ce type de modélisation ne réussit que dans des cas très favorables. En accord avec Durand-Guerrier (2007), nous considérons que les outils proposés par Brousseau - l'approche d'Alan Turing et celle de Paul Lorenzen - sont inadaptés à de nombreuses situations de validation.

Brousseau distingue trois types de situations adidactiques: les «situations d'action " dans lesquelles le milieu ne contraint pas les élèves à intégrer une verbalisation dans leurs stratégies, les «situations de formulation» dans lesquelles le milieu contraint cette fois les élèves à verbaliser et les « situations de validation» dans lesquelles le milieu contraint les élèves à confronter et à justifier leur verbalisation. Nous nous intéressons dans cet article plus spécifiquement aux situations de validation ${ }^{7}$. À propos de ces dernières, Brousseau affirme:

«Les situations de validation vont mettre en présence deux joueurs qui s'affrontent à propos d'un objet d'étude composé des messages et descriptions que l'élève a produits d'une part, et du milieu adidactique qui sert de référent à ces messages d'autre part (figure 7). Les deux joueurs sont alternativement un "proposant" et un "opposant"; ils échangent des assertions, des preuves et des démonstrations à propos de ce couple "milieu/ message" ». (Brousseau, 1998, p. 109)

Les situations de validation ont donc une triple dimension, une dimension syntaxique, une dimension sémantique et une dimension pragmatique (les messages et descriptions, la référence à ces messages, l'échange des assertions). L'objet d'étude pour celui qui s'intéresse à ces situations est un couple (un "proposant » et un "opposant ») et leurs échanges. En disant cela, Brousseau fait référence à la logique dialogique de Lorenzen dont il reprend le vocabulaire. Dans le paragraphe qui suit, nous présentons rapidement cette théorie. Nous montrerons ce qu'elle permet mais aussi en quoi elle est insuffisante pour modéliser une partie importante des situations de validation.

\section{La logique dialogique de Lorenzen}

À propos de l'assertion Vernant nous dit:

«De plus et surtout, l'analyse [de l'assertion] inaugurée par Frege, reprise par Austin, poursuivie par Searle et Vanderveken, se centre trop exclusivement sur l'acte du seul locuteur. Son défaut congénital est de rester monologique. Le «second "Wittgenstein a bien montré que les assertions ne pouvaient prendre sens que comme coups dans des jeux de langage qui se jouent au moins à deux. Et, avant même Wittgenstein, Peirce avait esquissé une analyse proprement dialogique de l'assertion. Ainsi, comme l'avait d'ailleurs bien compris Frege, l'assertion est réponse à une question antérieure. Lassertion doit donc s'analyser comme interacte prenant une fonction dialogique particulière. » (Vernant, 2005, p. 280 , traduction de Vernant)

Autrement dit, l'assertion doit être regardée comme une action, un coup, à l'intérieur d'un jeu et ne peut être analysée sans prendre en compte le jeu d'arrière-plan qui lui donne sens. En voici un 
exemple. Dans sa couverture du 11 décembre 2007 le journal gratuit Métro affirme:

« Alors que le conseil des ministres examine demain les mesures en faveur du pouvoir d'achat, $63 \%$ des Français préfèrent l'argent au temps libre et $78 \%$ se disent prêts à faire des heures sup. Lire p/04 » (nous soulignons)

Il nous semble qu'une manière " naturelle » de comprendre l'assertion ' 63 \% des Français préfèrent l'argent au temps libre'dans une perspective dialogique est que, à la question posée par un interlocuteur « préférez vous l'argent ou le temps libre? » (1), $63 \%$ des français interrogés répondent « l'argent ». Cette manière de comprendre l'assertion du journal Métro n'est cependant pas la bonne: la question réellement posée lors du sondage, que l'on peut trouver à la quatrième page de ce même journal, est « Si vous aviez le choix, que préfèreriez-vous...? Gagner moins d'argent et avoir plus de temps libre ou gagner plus d'argent mais avoir moins de temps libre » (2). Lorsqu'il est affirmé que '63\% des Français préfèrent l'argent au temps libre', il faudrait donc comprendre que les sondés ont choisi à $63 \%$ la deuxième réponse à la question (2). Les questions (1) et (2) n'étant clairement pas équivalentes, l'interprétation construite sur (1) constitue une mécompréhension de l'assertion en jeu.

L'objectif d'une analyse dialogique est de rechercher la fonction dialogique, de l'assertion afin d'éviter ce type de mécompréhensions ${ }^{8}$. La dimension dialogique de l'assertion est prise en compte dans la théorie des situations (les joueurs échangent, dit Brousseau) notamment en référence à Lorenzen dont nous présentons ici la logique dialogique. Nous essaierons de montrer que les préoccupations fondationnelles de Lorenzen l'ont conduit à proposer une théorie logique dialogique avec une certaine dimension pragmatique. À ce propos, Vernant affirme:

« La novation [celle de la logique dialogique de Lorenzen] s'avère essentiellement philosophique. Est introduite explicitement la dimension pragmatique en logique. Alors qu'en logique standard, la proposition excluait toute dimension énonciative pour se réduire à un simple porteur de valeur de vérité, en logique dialogique chaque proposition est véritablement une proposition émanant d'un interlocuteur qui s'engage sur elle par un acte d'assertion. » (Vernant, 2004, p. 9)

La logique dialogique de Lorenzen est un système formel dont l'objectif est de proposer une définition de la notion de vérité logique. Ce travail conduit Lorenzen à critiquer la logique classique. Il est selon lui pour le moins douteux d'appliquer la logique classique à des propositions dont la valeur de vérité ne peut pas être vérifiée (Lorenzen, 1967, p. 19). De son point de vue, il n'est par exemple pas raisonnable de considérer la proposition « Il y a des nombres impairs qui sont parfaits ${ }^{* 9}$ ou aucun nombre impair n'est parfait » comme une vérité logique si l'on ne dispose d'aucun procédé pour décider si « Il y a des nombres impairs qui sont parfaits » ou « Aucun nombre impair n'est parfait » en est une ${ }^{10}$. La conception de la vérité qu'il oppose est une théorie vérificationniste de la vérité. Pour chaque proposition, ce qui importe n'est pas de supposer qu'elle possède une valeur de vérité mais d'être capable de reconnaître une preuve de cette proposition lorsque celle-ci se présente. Lorenzen appelle les propositions pour lesquelles nous savons identifier une preuve, les propositions $p$-définies. Pour revenir à notre exemple, alors que l'on ne sait pas si elle est vraie ou fausse, la proposition « Il y a des nombres impairs qui sont parfaits » est $p$-définie puisque nous savons en identifier a priori une preuve (une preuve de cet énoncé s'identifie à la donnée d'un nombre impair parfait). Il est clair que les disjonctions finies ou infinies de propositions $p$-définies, les conjonctions finies de propositions $p$-définies sont des propositions $p$-définies. Par exemple « Il y a des nombres impairs qui sont parfaits ou aucun nombre impair n'est parfait » est $p$-définie puisque « Il y a des nombres impairs qui sont parfaits » et « Aucun nombre impair n'est parfait » le sont. Cependant, une conjonction infinie de propositions $p$-définies ou la négation d'une proposition $p$-définie ne sont pas automatiquement $p$-définies. Afin d'obtenir un ensemble clos sous l'action des constantes logiques*, Lorenzen est finalement amené à introduire la notion de propositions d-définies (dialogisch-definit). Celle-ci donne naissance à une explication dialogique et pragmatique de la validité:

«D'une façon générale, une proposition sera dite $d$ définie si, pour la soutenir dans un dialogue, les règles des deux partenaires sont déterminées de telle sorte qu'on peut décider à chaque instant si le dialogue est terminé et qui, dans ce cas, a gagné. » (Lorenzen, 1967, p. 21)

Cela permet à Lorenzen de traiter des conjonctions infinies, des négations et des implications. En effet, les conjonctions infinies de propositions $d$-définies, les négations de propositions $d$-définies, les implications 
construites à partir de propositions $d$-définies et du conditionnel, sont, comme nous pourrons le vérifier à partir des règles ci-dessous, des propositions $d$-définies. À partir de là, la vérité logique (la validité) effective peut être définie, elle fait intervenir des dialogues entre un proposant qui défend une proposition et un opposant qui tente de la falsifier:

« Nous dirons qu'une forme propositionnelle est logiquement vraie de manière effective si et seulement s'il est possible de gagner dans un dialogue toute proposition de cette forme (contre tout opposant).» (op. cité, p. 23)

Nous présentons maintenant les règles qui gouvernent le déroulement pas à pas du dialogue associé à l'évaluation de la validité d'une proposition et qui en assurent la cohérence logique. Les coups à l'intérieur $\mathrm{du}$ jeu dialogique sont réglementairement indépendants mais, comme les mouvements dans un jeu d'échec, ils peuvent être stratégiquement organisés ( $A_{1}$ et $A_{2}$ sont des propositions et $A$ un prédicat).

- Si un joueur ${ }^{11}$ soutient $A_{1} \wedge A_{2}^{*}$ l'autre joueur peut attaquer la conjonction en faisant un choix entre $A_{1}$ et $A_{2}$. La défense pour le premier joueur consiste alors à défendre l'assertion choisie par le joueur qui attaque.

- Si un joueur soutient $A_{1} \vee A_{2}{ }^{*}$, l'autre joueur peut attaquer la disjonction. Le défenseur choisit alors l'assertion qu'il souhaite défendre (entre $A_{1}$ et $A_{2}$ ).

- Si un joueur soutient $\forall \mathrm{xA}(\mathrm{x})^{*}$, l'autre joueur peut attaquer l'énoncé universel. Le défenseur défend alors l'assertion $A(t)$ où la lettre $t$ est choisie par l'attaquant.

- Si un joueur soutient $\exists \mathrm{xA}(\mathrm{x})^{*}$, l'autre joueur peut attaquer l'énoncé existentiel. La défense consiste alors à choisir une lettre $t$ et à faire l'assertion $A(t)$.

- Si un joueur soutient $A_{1} \Rightarrow A_{2}{ }^{*}$, l'autre joueur peut attaquer le conditionnel en engageant un sous jeu autour de la défense de l'assertion $A_{1}$. Si le joueur qui soutenait $A_{1} \Rightarrow A_{2}$ ne parvient pas à gagner ce sous jeu, il peut continuer à se défendre par l'assertion $A_{2}$.

- Si un joueur soutient $\neg A_{1}{ }^{*}$, l'autre joueur peut attaquer par l'assertion $A_{1}$.

Durant le jeu, si le proposant souhaite soutenir une proposition atomique ${ }^{*}$, il doit s'assurer que celle-ci ait préalablement été soutenue par l'opposant. Cette restriction ne s'applique pas à l'opposant. La raison de cette dissymétrie est que la logique dialogique de Lorenzen est une théorie "syntaxique " de la vérité: seule la forme des énoncés doit être utilisée pour s'assurer de leur validité. Le jeu se termine lorsqu'un des joueurs ne peut plus avancer d'arguments, ce joueur a alors perdu le dialogue. Dire qu'un énoncé est valide c'est selon Lorenzen dire qu'il existe une stratégie qui permette au proposant de gagner le dialogue quels que soient les choix de l'opposant et nous disposons d'une démonstration si nous connaissons une telle stratégie de gain. Voici un exemple emprunté à Lorenzen:

\begin{tabular}{|c|c|c|l|}
\hline & Opposant & Proposant & \multicolumn{1}{c|}{ Commentaires } \\
\hline 1 & & $\mathrm{a} \vee \neg \mathrm{a}$ & $\begin{array}{l}\text { Le proposant soutient le principe du tiers exclu selon lequel la } \\
\text { disjonction formée d'un énoncé et de sa négation est valide. }\end{array}$ \\
\hline 2 & $?$ & $\neg \mathrm{a}$ & $\begin{array}{l}\text { Lopposant attaque la disjonction (ceci est symbolisé par ?), le proposant } \\
\text { choisit alors de se défendre par } \neg \text { a (il n’a pas le choix puisque les règles } \\
\text { du jeu lui interdisent de défendre la proposition atomique } a)\end{array}$ \\
\hline 3 & $\mathrm{a}$ & Lopposant répond par a (règle de la négation) \\
\hline
\end{tabular}


Le proposant a perdu le dialogue puisqu'il n'a rien à répondre à la dernière assertion de l'opposant en (3). Le proposant n'ayant pas d'autre choix que de soutenir $\neg$ a en (2), cette défaîte n'est pas accidentelle. Ceci montre que la proposition a $\vee \neg$ a n'est pas logiquement valide au sens de Lorenzen car le proposant ne dispose pas d'une stratégie gagnante (pour cela, il lui faudrait être en mesure de gagner tous les dialogues, quels que soient les choix de l'opposant).

Lorenzen donne comme règle le fait que le proposant ne peut pas soutenir une proposition atomique à moins que l'opposant ne l'ait déjà soutenue. Cependant, ce modèle ne tient pas compte de la possibilité, affirmée par Brousseau, d'échanger des preuves et des démonstrations ou (cf. ci-dessous $\S 4$ ) des vérifications sémantiques. Il est possible de tenir compte des échanges de démonstration par une modification de la règle précédente en autorisant le proposant à soutenir des propositions dont il connaît une démonstration (une stratégie gagnante dans le jeu dialogique associé) sans que l'opposant ne puisse la remettre en cause. Pour tenir compte des vérifications sémantiques, il faudrait assouplir la règle concernant les propositions atomiques en permettant au proposant de soutenir une proposition atomique même si l'opposant ne l'a pas déjà soutenue, dans le cas où les joueurs parviennent à un accord sur sa vérité. Dans ce cas, la forme des énoncés n'intervient plus seule dans la recherche de la vérité, des éléments extérieurs au dialogue interviennent et une théorie sémantique est nécessaire. Il nous semble que les règles de la logique dialogique de Lorenzen, ou une version avec des règles du jeu amendées, permettent de rendre compte du fonctionnement de ce que Wittgenstein appelle les convictions inébranlables:

«Et maintenant si je disais: « c'est ma conviction inébranlable que, etc », cela veut dire, dans le cas présent aussi, que je ne suis pas parvenu à cette conviction consciemment en suivant un processus de pensée déterminé, mais qu'elle est à point ancrée dans toutes mes questions et réponses que je ne peut pas y toucher. »(Wittgenstein, 1976, § 103)

Cependant, si dans le dialogue une proposition nécessite l'intervention d'un tiers, ce processus externe ne peut être pris en compte par le système que nous avons présenté. La théorie dialogique et sémantique de Hintikka revendique le traitement des « jeux d'extérieur» (des jeux qui prennent en compte les objets qui sont extérieurs au langage) qu'il oppose aux « jeux d'intérieur » décrits ci-dessus (Hintikka, 2007, p. 67). Nous la présenterons après avoir montré un exemple concret d'usage de la logique dialogique à travers la modélisation d'un dialogue d'« intérieur».

Exemple 1: Une modélisation d'un dialogue autour de la rationalité de $\sqrt{2}$ s'appuyant sur la logique dialogique

Les transcriptions que nous allons étudier dans cet article sont issues du corpus de thèse de Battie ${ }^{12}$. Dans cette recherche, Battie étudie l'hypothèse, qui semble fonder le retour de l'arithmétique dans les programmes français, selon laquelle l'arithmétique est un lieu privilégié pour le travail du raisonnement mathématique. Les extraits que nous allons étudier proviennent de la transcription des dialogues d'un groupe de trois élèves (le groupe A de son expérimentation) de terminale $S$ (option mathématiques) concernant la rationalité de $\sqrt{2}$.

L'exercice proposé aux élèves:

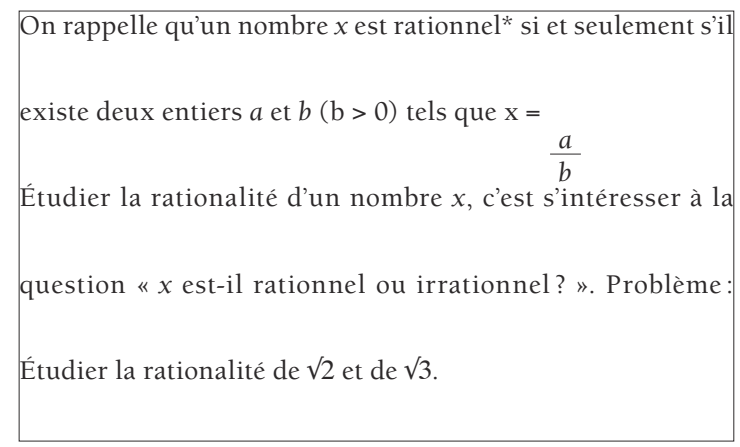

\section{Analyse a priori}

Cette analyse s'appuie sur les travaux de Battie (Battie, 2003, 2007). Nous nous concentrons sur l'étude par les élèves de la rationalité de $\sqrt{2}$ car les extraits qui nous intéressent proviennent de cette étude. Battie distingue et explicite six preuves disponibles pour les élèves de ce niveau (la preuve fondamentale, la preuve originale, la preuve classique, la preuve par descente infinie, la preuve par minimalité et la preuve par récurrence). Nous restreignons notre analyse a priori à la description des preuves « originale » et « classique». 
Cette description nous semble suffisante pour éclairer les courts extraits que nous avons choisis.

Une écriture possible de la «preuve classique » est:

« On suppose qu'il existe deux entiers naturels premiers entre eux ${ }^{*}$ dont le quotient vaut $\sqrt{ } 2$. Soient $a$ et $b$ deux tels entiers, on $a_{b}^{a}=\sqrt{2}$. En prenant le carré, on obtient $a^{2}=2 b^{2}$ (1) ce qui montre que $a^{2}$ est multiple $e^{*}$ de 2 et donc que $a$ est multiple de 2 (2). On peut donc écrire $a=2 k$ pour un certain entier naturel $k$. En injectant cette écriture dans (1), on obtient les égalités suivantes: $(2 k)^{2}=2 b^{2} \Leftrightarrow 2^{2} k^{2}=2 b^{2} \Leftrightarrow 2 k^{2}=b^{2}$. Ceci montre que $b^{2}$ est multiple de 2 et donc que $b$ est multiple de 2 (3). (2) et (3) sont incompatibles avec le fait que $a$ et $b$ sont premiers entre eux, ceci montre que l'hypothèse de départ est fausse et donc que $\sqrt{2}$ est irrationnel. »

Cette preuve par l'absurde est celle qui domine institutionnellement. Il s'agit de la preuve attendue par l'enseignante, d'autant plus qu'elle l'a déjà présentée à la classe à l'occasion du cours d'arithmétique.

Une écriture possible de la «preuve originale » est:

« On suppose qu'il existe deux entiers naturels premiers entre eux dont le quotient vaut $\sqrt{2}$. Soient $a$ et $b$ deux tels entiers, on a donc $\frac{a}{b}=\sqrt{ } 2$. En prenant le carré, on obtient $a^{2}=2 b^{2}$ ce qui montre que $a^{2}$ divise $2 b^{2}$. Mais $a^{2}$ et $b^{2}$ sont premiers entre eux puisque $a$ et $b$ le sont et donc d'après le théorème de Gauss ${ }^{*} a^{2}$ divise 2. Ceci montre que $a=1$ puisque les seuls diviseurs de 2 sont 1 et 2 . La contradiction vient du fait qu'il est impossible que $\frac{1}{b}=\sqrt{ } 2$ puisque par exemple $\frac{1}{b} \leq 1$, l'hypothèse de départ est donc fausse ce qui permet de conclure que $\sqrt{2}$ est irrationnel. »

La preuve originale se distingue de la preuve classique notamment par l'usage du théorème "Soit $a$ et $b$ deux entiers naturels, on a alors $(\operatorname{pgcd}(a, b)=1) \Rightarrow\left(\operatorname{pgcd}\left(a^{2}, b^{2}\right)=1\right) »$

Ce théorème ne fait pas partie du bagage institutionnel de l'arithmétique. D'autre part, cette preuve fait aussi intervenir la divisibilité par 1, laquelle ne vit que rarement dans l'enseignement.

\section{Les Extraits}

Chacun des extraits que nous utiliserons dans cet article provient de la transcription des dialogues d'un même groupe. Nous décrivons ici la trame des dialogues concernant la rationalité de $\sqrt{2}$ en réutilisant le découpage proposé par Battie (2003, chapitre 8, II. 2) et nous y situons les extraits que nous analysons.

Épisode 1. Les élèves engagent le problème en écrivant l'égalité $a=b \sqrt{ } 2$ et se posent des questions quant aux conséquences en terme de divisibilité de cette égalité (il semble procéder comme si $\sqrt{2}$ était entier). Finalement, l'idée d'une preuve par l'absurde émerge.

Épisode 2. Une nouvelle piste est explorée. Elle consiste à mettre l'égalité $a=b \sqrt{2}$ au carré et à essayer de construire un raisonnement par parité. La piste est abandonnée.

Épisode 3. Le groupe travaille à nouveau autour de l'égalité $a=b \sqrt{ } 2$ mais la recherche ne semble pas aboutir.

Épisode 4. L'enseignante intervient pour confirmer qu'il est préférable de travailler sur l'égalité $a^{2}=2 b^{2}$. Le travail s'oriente alors autour de la question de la validation de la proposition, essentielle dans la preuve originale, qui affirme que pour tout couple d'entiers naturels, si ils n'admettent que 1 comme diviseurs commun alors il en est de même de leur carré: $\forall a \forall b(\operatorname{pgcd}(a, b)=1) \Rightarrow\left(\operatorname{pgcd}\left(a^{2}, b^{2}\right)=1\right.$. Un extrait de cet épisode sera analysé dans le paragraphe 3 de cet article et un autre dans le paragraphe 4 .

Épisode 5. L'enseignante intervient à nouveau pour montrer comment utiliser le fait, reconnu par les élèves, que $a^{2}$ est pair. Cette partie de l'épisode sera analysée dans le paragraphe 4 de cet article. Les apports de l'enseignante permettent à une élève de parvenir à une solution (il s'agit de la preuve classique).

Épisode 6. À la demande de l'enseignante, l'élève qui est parvenue à construire la preuve classique explique sa démarche à une autre élève du groupe. Nous analysons maintenant un extrait de cet épisode. 
Extrait issu de l'épisode 6

0 . Alors je t'explique. Tu as compris jusqu'à/

1. Parle doucement. (rires)

2. T'as compris jusqu'à là que $a$ est multiple de $2 /$

3. Oui, ça j’ai compris.

4. T'as a est multiple de 2, d'accord? ? donc que $a^{2}$ est égal à $2 b^{2} /$

5. Non mais j'ai compris que $b$ était multiple de 2 .

6 . Ben voilà... Donc $a$ multiple de 2 donc $a$ égal

$2 q$ et $b$ égal $2 q^{\prime}$, t'es d'accord? Les deux sont multiples de 2. Quand tu fais $a$ sur $b$, et ben cette fraction est réductible* puisque tu peux la réduire par 2 .

7. Et alors?!

8. Donc c'est pas possible parce que euh, $x$, euh si racine de 2 s'écrit $a$ sur $b$ il faut que $a$ et $b$ et bien ils soient premiers entre eux. T'es d'accord?

9. Ben non ces deux-là ils sont premiers entre eux maintenant.

10. Mais non $a$ égal $2 q$ et $b$ égal $2 q$ '

11. Oui!

12. Donc et ben $a$ et $b$ ils sont pas premiers entre eux

13. Ca fait $q$ sur $q$ '

14. On s'en fout de $q$ sur $q$ '.

15. Ben si.

16. -tends, regarde. Ah!

17. Attends mais c'est la même chose!

18. Chut! Tu me laisses parler, tu me laisses parler

OK? Bon. Tu dis, est-ce que ça va si racine de 2 il

peut s'écrire $a$ sur $b$ irréductible (elle insiste sur ce mot). D'accord?

19. C'est bon me regarde pas comme ça. (rires)

20. Le problème, tu veux savoir si racine de 2 il peut s'écrire avec $a$ sur $b$ avec $a$ et $b$ irré enfin/

21. Ils sont premiers entre eux.

22. Premiers entre eux. Voilà. pgcd $(a, b)$ égal 1 , d'accord? Voilà. Or on a fait tout un bidouillage, on a trouvé que $a$ égal $2 q$ et $b$ égal $2 q$ '. Or on a dit que pgcd égal 1 donc là ils ont 2 en commun donc c'est pas possible, donc racine de $2 /$

23. D'accord, j'ai compris!

24. s'écrit pas sur $a$ sur $b$ donc racine de 2 est irrationnel.

25. Voilà.

26. Voilà, maintenant tu rédiges. (Rires)

27. C'est toi qui rédiges, c'est la prof qu'a dit que tu rédiges.
Analyse

-Première partie: Une théorie locale

Nous analysons dans ce paragraphe les interventions des élèves de (0) à (17). Notre analyse décrit l'émergence de normes, organisées en une théorie locale, qui régissent le déroulement du discours. Dans cette modélisation, le proposant modélise l'élève qui explique sa preuve et l'opposant celui qui ne l'a jusque-là pas comprise. Cependant, nous ne prétendons pas qu'il soit possible de faire correspondre chaque assertion des élèves avec les assertions de leur modèle.

Axiomes ${ }^{13}$

$a=2 q, b=2 q^{\prime},\left(a=2 q \wedge b=2 q^{\prime}\right) \Rightarrow \neg(\operatorname{pgcd}(a, b)$

$=1, \operatorname{pgcd}(a, b)=1)$

\begin{tabular}{|c|c|c|}
\hline & Opposant & Proposant \\
\hline A & & $\left(\mathrm{a}=2 \mathrm{q} \wedge \mathrm{b}=2 \mathrm{q}^{\prime}\right)$ \\
\hline B & $\neg(\operatorname{pgcd}(a, b)=1)$ & \\
\hline C & & $\operatorname{pgcd}(a, b)=1$ \\
\hline
\end{tabular}

Commentaires:

Le début du dialogue consiste en une mise en place conjointe de la théorie, les deux premiers axiomes sont explicités de (0) à (5), le troisième en (6) et (7), le quatrième en (8) et (9). Ils font partie, avec les règles d'usage des constantes logiques, des règles du jeu qui est joué. Le proposant attaque alors le troisième axiome de la théorie en faisant l'assertion de l'antécédent de l'implication - (10) et $A$ - ce à quoi l'opposant rétorque par le conséquent - (12), (13) et $B$ - car il est inutile pour lui d'attaquer la conjonction puisque chacun de ses termes sont des axiomes (11). Le proposant peut attaquer en $C$ la négation $B$ puisque $C$ est un axiome de la théorie, ce à quoi l'opposant ne peut rien répliquer. Il gagne donc le dialogue ce qui montre que la théorie est contradictoire car le proposant dispose d'une stratégie gagnante d'attaque d'un des axiomes de la théorie. Dans la suite du dialogue, le proposant va essayer de montrer qu'il dispose d'une stratégie qui montre que l'hypothèse de rationalité de $\sqrt{ } 2$ implique les axiomes de la théorie locale précédente et donc qu'il faut la rejeter. On peut cependant remarquer que 
dans ce premier temps du dialogue l'élève modélisée par l'opposant ne semble pas se rendre compte de la contradiction revendiquée par l'autre élève - (13), (15) et (17). De ce point de vue, notre modélisation rend certainement mieux compte de l'activité de l'élève qui expose sa preuve, que de l'activité de l'élève qui essaye de la comprendre ${ }^{14}$. Au niveau de la modélisation, l'explication de cette incompréhension semble se situer autour du quatrième axiome de la théorie que nous avons considéré comme structurant le jeu malgré l'ambiguité de (9). Il y a dans cette pratique une composante arbitraire puisque aucun élément expérimental ne nous permet d'affirmer qu'un élève suit effectivement un ensemble de règles (dont font partie ici, les axiomes de la théorie), et, a fortiori, que les deux interlocuteurs $\mathrm{du}$ dialogue suivent les mêmes règles ${ }^{15}$. Malgré cela, modéliser l'activité langagière de plusieurs interlocuteurs à l'intérieur d'une même structure logique peut permettre d'interpréter les incompréhensions.
-Deuxième partie: l'hypothèse de rationalité est contradictoire

Ce paragraphe a pour objet l'analyse des assertions (18) à (24). Le proposant et l'opposant continuent de modéliser les mêmes élèves. Dans cette deuxième partie de dialogue, le proposant, selon notre modélisation, entreprend de monter qu'une théorie conjointement admise comprenant l'hypothèse de rationalité de $\sqrt{2}$ implique la théorie précédente dont il a montré le caractère contradictoire.

Axiomes:

$$
\begin{aligned}
& \left(\sqrt{ } 2=\frac{a}{b}\right) \wedge(\operatorname{pgcd}(a, b)=1), \\
& \left(\sqrt{ } 2=\frac{a}{b}\right) \Rightarrow\left(\exists k, a=2 k \wedge \exists k^{\prime}, b=2 k^{\prime}\right), \\
& \left(a=2 q \wedge b=2 q^{\prime}\right) \Rightarrow \neg(\operatorname{pgcd}(a, b)=1)
\end{aligned}
$$

\begin{tabular}{|c|c|c|}
\hline & Opposant & Proposant \\
\hline A & $\left(\sqrt{ } 2=\frac{a}{b}\right) \wedge(p g c d(a, b)=1)$ & $? 1$ \\
\hline B & $p g c d(a, b)=1$ & $? 2$ \\
\hline C & $\sqrt{ } 2=\frac{a}{b}$ & $\sqrt{ } 2=\frac{a}{b}$ \\
\hline D & & $? 1 ? k$ \\
\hline E & & $? 2 ? k$, \\
\hline F & $a k, a=2 k \wedge \exists k, b=2 k^{\prime}$ & \\
\hline G & $b=2 q$ & \\
\hline
\end{tabular}

\section{Commentaires:}

Le premier axiome est établit en (18), (19), (20) et (21) les deux suivants en (22). Le proposant va essayer de montrer que cette théorie implique la théorie précédente. Il attaque en $A$ et $B$ le premier axiome en demandant à l'opposant de soutenir chacun des termes de la conjonction celui-ci le fait en $B$ et $C-(21)$ et (22). Le proposant peut alors attaquer en $D$ l'antécédent du deuxième axiome ( « tout un bidouillage ») puisque cette proposition atomique a été défendue par l'opposant en C. L'opposant répond alors en $E$ par le conséquent puis concède les deux premiers axiomes de la théorie locale précédente ( $F$ et $G$ ). En (22) et (23) le constat est fait que la théorie considérée implique la théorie locale qui émerge des assertions (0) à (17) (les axiomes de cette théorie sont soit des axiomes de cette nouvelle théorie soit des assertions - B, F et $G$ - de l'opposant) et donc que l'hypothèse de rationalité de $\sqrt{ } 2$ doit être rejetée. Cette fois-ci, l'élève qui se faisait expliquer la preuve dit avoir compris l'argumentation de son interlocutrice, ce qui implique probablement qu'elle a du relever le lieu de la contradiction et en particulier accepter l'axiome $\operatorname{pgcd}(a, b)=1$ qui jusque-là posait problème. Dans la modélisation ceci peut s'interpréter par le fait 
que l'opposant est contraint de faire l'assertion $\mathrm{pgcd}$ $(a, b)=1$ en $B$ et donc qu'il est contraint de l'assumer alors que dans la modélisation de la première phase du dialogue l'opposant ne l'avait pas été ${ }^{16}$.

\section{Conclusion:}

À travers cet exemple, notre objectif était de montrer la possibilité d'un usage de la logique dialogique à des fins modélisatrices dans le contexte particulier qui est celui d'une explication de raisonnement. Si la référence théorique de Brousseau à Lorenzen semble bien connue, nous ne connaissons pas de travaux de recherche en didactique des mathématiques qui en fassent un usage explicite. Pourtant Lorenzen lui-même revendique une certaine proximité entre les règles de sa logique dialogique et des pratiques plus profondes. Selon Marion:

"This is why Lorenzen argues that his rules are abstracted from what he called our 'nonverbal activity'(die Praxis unseres sprachfreien Handelns) or our 'prelogical speech practice'(vorlogische Redpraxis) [...]. » (Marion, 2006b, p. 11)

Nous allons maintenant essayer de montrer que le point de vue sémantique apporté par Hintikka sur les dialogues s'avère plus efficace lorsqu'il s'agit de procéder à des modélisations de phase de recherche.

\section{La sémantique GTS de Hintikka et la correction de Vernant}

« En fait, on peut considérer la sémantique des jeux comme une codification de la manière de penser et de parler que les mathématiciens (et les spécialistes de logique mathématique) utilisent depuis des temps immémoriaux (au moins depuis le temps de Cauchy) mais qu'ils n'ont reconnue en tant que outil conceptuel que lorsque les autres moyens qu'ils avaient de traiter leurs sujets ont cessé de remplir leur office. » (Hintikka, 1994, p. 142)

Selon Hintikka, la sémantique GTS peut être vue comme " une codification de la manière de penser et de parler des mathématiciens ", il dira même ailleurs que (Hintikka, 1996, p. 98) « la logique reflète les caractéristiques structurelles de nos activités de recherche et de résolution ». Il y a chez lui une volonté de rendre compte, à la manière de Kant (Hintikka, 1996), de l'activité mathématique. Il défend en particulier l'idée que sa sémantique GTS constitue le "médium », le point de rencontre, entre la réalité extérieure et notre connaissance mathématique de cette réalité. Les jeux « de recherche et de résolution » dont se préoccupe Hintikka sont donc des jeux d'extérieur dans le sens où ils mettent en œuvre des manipulations d'objets extra-langagiers là où la logique dialogique de Lorenzen manipule des symboles du langage. Cette distinction se retrouve à travers une variation de vocabulaire puisque Hintikka utilise les termes de Moi-même et de Nature à la place des termes de Proposant et Opposant. Selon lui, l'erreur de Lorenzen est de s'être concentré sur des jeux d'intérieur, des jeux formels, indépendants de l'expérience alors que la démarche rationnelle de recherche fait intervenir des données extérieures au langage.

«In the approach I am criticizing, a yet different answer is, in effect, given to the same question. It amounts to identifying formal argumentation as the relevant kind of knowledge-acquiring activity. " (Hintikka \& Kulas, 1983, p. 39)

Cependant, comme Vernant le fait remarquer, la Nature n'intervient pas dans les activités de recherche et de résolution comme un interlocuteur mais plutôt comme un arbitre (dans un contexte didactique, le rôle de la Nature peut être joué par l'enseignant, le livre, la calculatrice,...):

« La découverte de la pesanteur de l'air par Pascal et Torricelli ne s'est pas faite en dialoguant avec Dame Nature, mais en inventant une hypothèse, en élaborant un protocole expérimental et en faisant effectuer la vérification au sommet du Puy de Dôme. Par contre, dans le dialogue épistolaire entre Pascal et Torricelli, la Nature intervenait constamment en tiers venant sanctionner à travers l'enquête expérimentale, les hypothèses proposées. Hintikka a bien vu la nécessité d'articuler l'interaction dialogique sur une transaction intramondaine, mais il a manifestement confondu fonction dialogique et procédure de vérification. La nature n'est pas l'opposant, mais le tiers qui sanctionne, l'autorité à laquelle se plient les savants. »(Vernant, 2004, p. 13)

Nous utiliserons à la suite de Lorenzen, Brousseau et Vernant les termes de Proposant et Opposant pour désigner les interlocuteurs du dialogue. Les règles du jeu de la sémantique GTS sont des adaptations sémantiques de celles de la logique dialogique. En particulier, les règles qui traitent des quantificateurs ou des propositions atomiques sont corrigées. Pour ce qui 
est des règles structurelles, les variations par rapport au système de Lorenzen sont aussi les conséquences de l'approche sémantique de Hintikka. À l'image de l'explication sémantique de la notion de vérité de Tarski, Hintikka utilise les notions de « domaine d'objets » et d' « interprétation». Pour associer un jeu à une proposition, il faut tout d'abord interpréter chaque constante extra-logique* dans cette structure d'interprétation. Ensuite, chaque énoncé atomique et chaque identité formée à partir du langage considéré et d'un ensemble fini de variables, reçoit une valeur de vérité. Le fait que les jeux considérés soient des jeux sur des domaines d'objets permet une modification des règles de manipulation des quantificateurs. Plus exactement, alors que dans la logique dialogique ces choix sont des choix formels, des choix de lettres où la liberté de l'interlocuteur consiste à décider si il choisit une lettre déjà introduite (dans les cas où c'est possible) ou non, les choix de la sémantique des jeux consistent en de véritables sélections d'objets parmi le domaine d'objets (la structure interprétative). D'autre part, étant donné que chaque proposition atomique est interprétée et qu'elle a reçu une valeur de vérité (le jeu est à information complète), le proposant peut, comme l'opposant, soutenir une proposition atomique même si l'opposant ne l'a pas préalablement fait.

Un énoncé est dit vrai (dans une structure d'interprétation) si il existe une stratégie gagnante pour le proposant contre n'importe quel opposant sur le jeu associé à l'énoncé et à l'interprétation sur le domaine d'objets. La validité logique d'un énoncé peut alors se définir comme la vérité pour toutes les interprétations possibles sur des domaines d'objets. Une de nos hypothèses est que cette notion formelle de "stratégie " puisse contribuer à une explication de la notion informelle de «stratégie » qui se situe au fondement épistémologique de la théorie des situations (§ 1). En particulier, la modélisation en termes de stratégie permet d'expliciter la correspondance avancée par Wittgenstein entre règle et signification (cf. introduction). Un apprentissage peut se comprendre comme le processus de conformation aux règles stratégiques d'usage des termes qui constituent leur signification dans la communauté mathématique.

Durand-Guerrier（Durand-Guerrier, 2007, p. 22) affirme l'intérêt didactique de la démarche de Vernant. Celui-ci met en effet en perspective l'ap- proche sémantique de Tarski, dont elle a montré la pertinence didactique, avec la logique dialogique de Lorenzen à travers une correction de la sémantique GTS de Hintikka. Nous décrivons ici cette correction puisqu'elle nous paraît adaptée à un usage didactique de la sémantique GTS.

L'hypothèse selon laquelle les jeux structurant les processus de recherche de la vérité se faisaient contre la Nature - un joueur omniscient - a conduit Hintikka à considérer que ces jeux étaient des jeux à information complète (que les valeurs de vérité de toutes les propositions atomiques étaient connues des joueurs). D'autre part, c'est une condition vraisemblablement nécessaire à son entreprise fondationnelle:

«Ce que fait ma caractérisation, c'est qu'elle étend la notion de vérité [à partir de celle des propositions atomiques] à tous les autres énoncés du langage en question. Le fait que je restreigne ainsi ma tâche ne signifie pas que je ne pense pas nécessaire d'étendre l'analyse modèle-théorétique des significations. Si j'impose cette restriction, c'est parce que sans elle la portée de mon entreprise deviendrait trop large, irréaliste et ingérable. » (Hintikka, 2007, p. 55)

Si nous nous intéressons aux dialogues entre élèves, nous ne pouvons pas supposer que chaque proposition atomique a reçu une valeur de vérité. Le plus souvent, ces dialogues sont à information incomplète, c'est-à-dire que bien que chaque proposition atomique soit interprétée dans un domaine d'objet, les interlocuteurs ne sont pas capables de donner systématiquement une valeur de vérité aux propositions atomiques. Celles-ci sont parfois évaluées (vrai, faux ou inconnu) dans le cours du jeu, notamment à travers l'intervention d'un tiers (la nature, l'expérience, le livre, l'ordinateur, le professeur,...). La correction prend la forme suivante (Vernant, 2004, p. 14):

« RS 1'- Les propositions atomiques assertées par chaque interlocuteur sont vérifiées par une procédure transactionnelle acceptée conjointement par les deux interlocuteurs (chaque proposition est alors admise pour vraie, fausse ou de valeur inconnue).»

Cette version corrigée s'écarte définitivement des perspectives fondationnelles de Lorenzen ou Hintikka pour une perspective pragmatique puisqu'il se peut que des jeux se terminent sans vainqueur (par exemple dans le cas où les joueurs ne parviennent pas à se mettre d'accord sur la valeur de vérité d'une 
proposition atomique) ou alors qu'ils se terminent par la victoire d'un joueur qui n'aurait pas dû gagner (par exemple si il y a erreur dans l'évaluation d'une proposition atomique).

Nous proposons maintenant un exemple d'utilisation de cette correction de la sémantique GTS.

\section{Exemple 2: Une modélisation d'un dialogue s'appuyant sur la sémantique GTS}

Nous utilisons à nouveau le corpus de Battie (Battie, 2003). Le dialogue ci-dessous correspond à une partie de l'épisode 4. Au début de cet épisode, l'enseignante invalide la manipulation des concepts de divisibilité à partir de l'égalité $a=b \sqrt{ } 2$ et oriente le travail du groupe vers l'égalité $a^{2}=2 b^{2}$. Elle poursuit son intervention avec pour objectif de mettre les élèves sur la voie de la preuve classique. Les élèves ne la suivront cependant pas. Une question émerge: le théorème $\forall a \forall b(\operatorname{pgcd}(a, b)=1) \Rightarrow\left(\operatorname{pgcd}\left(a^{2}, b^{2}\right)\right.$ $=1)$ est-il valable ${ }^{17}$ ?

\section{Un extrait issu de l'épisode 4}

0 . N'oublie pas qu'on a a et b premiers entre eux hein. $\mathrm{a}^{2}$ est premier à $\mathrm{b}^{2}$ aussi.

1. Pas obligé.

2. Hum, j'en suis pas très sûre.

3. Attends on va prendre un exemple. 3 est premier avec

2 donc 9 est premier avec 4 .

4. Ouais mais bon...

5. J'sais pas si ça marche inaudible

6. Al: Regarde 2 et 5 ils sont premiers entre eux. Non, j'ai rien dit.

(Rires)

7. 9 t'as qu'à prendre 9,9 et 17 . Mais moi j'connais pas le carré de 17.

8. Al : J'ai une calculatrice (en rigolant).

9. Vas-y fait, fait 17 au carré divisé par 9 au par 81 .

10. Al: Oui mais c'est deux nombres premiers* faudrait prendre/

11. Oui ben c'est ce qu'on a dit, on a dit des nombres premiers.

12. Entre eux.

13. À: Mais non! Premiers entre eux pas premiers Ah ouais d'accord.

14. Al: $4^{2}$ et j'sais pas et euh/
15. J'sais pas prends 15 et euh 15 et?

16. À: 15 et 4 j'ai mis.

17. Ben c'est pareil.

18. À: Ou 125 et 16 . Ils sont premiers entre eux.

19. J'en sais rien moi.

(Rires)

20. Tu mets 125 divisé par 16 tu verras bien... Non c'est pas comme ça qu'on fait. 16 par 16 c'est quatre 2 , 2 fois $2 /$

21. A: Non moi j'crois qu'ils sont premiers entre eux, 16 et 125 .

22. Ouais quand on met les trucs au carrél

23. À: Ouais mais on sait pas, c'est pas écrit dans le cours mais on peut pas le démontrer dans le cas général/ 24. Oh on s'en fout!

25. Al : Donc on n'a pas le droit de l'utiliser. Enfin j'crois pas, j'sais pas peut-être qu'on l'a dit dans le cours

Analyse

La recherche étant menée de manière conjointe par les trois élèves du groupe, le proposant et l'opposant ne modélisent pas d'élèves en particulier. Chaque élève peut être tour à tour dans le rôle du proposant ou de l'opposant. Le phénomène est d'ailleurs accentué par le fait que les élèves jouent plusieurs fois le même jeu afin vraisemblablement d'une part de se convaincre de la validité du théorème et d'autre part de susciter l'émergence d'une stratégie de preuve. Une modélisation dialogique nous semble néanmoins pertinente. Comme le fait remarquer Lorenz, continuateur des travaux de Lorenzen, l'intérêt de l'analyse dialogique ne se limite pas au cas où les interlocuteurs sont clairement distingués:

«Quand un agent accomplit une action, il est aussi en possession d'une « image » de son action (il se voit agir), et de façon similaire, un locuteur qui parle de quelque chose est aussi, en disant, en possession de ce qu'il/elle "signifie » (il/elle agit toujours comme son propre auditeur). [...]. Cependant, à être pleinement conscient, on peut transformer de telles différences en des processus d'apprentissage de chacun [...]. » (Lorenz, 2003, p. 59-60)

Dans le tableau ci-dessous, chacune des quatre colonnes qui scindent les colonnes « Opposant » et "Valeur de vérité », correspond à une partie du jeu sémantique. 


\begin{tabular}{|c|c|c|c|c|c|c|c|c|c|}
\hline & \multicolumn{4}{|c|}{ Opposant } & Proposant & \multicolumn{4}{|c|}{ Valeur de vérité } \\
\hline A & & & & & $\forall a \forall b(\operatorname{pgcd}(a, b)=1) \Rightarrow\left(\operatorname{pgcd}\left(a^{2}, b^{2}\right)=1\right)$ & & & & \\
\hline B & $\begin{array}{l}a=2 \\
b=3\end{array}$ & $\begin{array}{l}a=2 \\
b=5\end{array}$ & $\begin{array}{c}a=9 \\
b \\
=17\end{array}$ & $\begin{array}{c}a=4 \\
b \\
=15\end{array}$ & $(\operatorname{pgcd}(a, b)=1) \Rightarrow\left(\operatorname{pgcd}\left(a^{2}, b^{2}\right)=1\right)$ & & & & \\
\hline $\mathrm{C}$ & \multicolumn{4}{|c|}{$\operatorname{pgcd}(a, b)=1$} & & vrai & vrai & ? & (vrai) \\
\hline D & & & & & $\operatorname{pgcd}\left(a^{2}, b^{2}\right)=1$ & vrai & $?$ & ? & $? /$ vrai \\
\hline
\end{tabular}

\section{Commentaires:}

Les joueurs sont en désaccord sur la validité de la proposition $A \forall a \forall b(\operatorname{pgcd}(a, b)=1) \Rightarrow\left(\operatorname{pgcd}\left(a^{2}, b^{2}\right)\right.$ =1) dont la structure d'interprétation est l'ensemble des entiers naturels, il y a donc un enjeu de vérité (0), (1) et (2). Ensuite, l'opposant fait des choix en $B$ d'éléments de la structure d'interprétation - (3), (6), (7), (14) et (15) - qui lui permettent d'attaquer l'antécédent de $B$ en $C$. La stratégie de l'opposant consiste à choisir des éléments qui rendent $C$ vrai (sinon il perd le dialogue). Le proposant répond alors par le conséquent. Les procédures transactionnelles consistent en des calculs (certains sont instrumentés, d'autres non). Certaines parties engagées sont écourtées et ne se terminent pas mais aucune partie ne se termine par la victoire de l'opposant ce qui conduit le groupe à faire l'hypothèse de la vérité du théorème (pour l'interprétation qui est faite). La décision semble prise mais le groupe n'entre pas dans le jeu de recherche d'une justification de la robustesse de la stratégie employée, c'est-à-dire dans la recherche d'une justification pour la vérité du théorème.

\section{L'insuffisance d'une modélisation fondée sur la}

\section{logique dialogique}

Dans ce paragraphe, nous envisageons quatre possibilités de modélisation fondées sur la logique dialogique en nous restreignant à l'analyse de ce que le point de vue sémantique qualifie de première et quatrième partie (en considérant que la procédure transactionnelle d'évaluation de $\operatorname{pgcd}\left(4^{2}, 15^{2}\right)=1$ a conduit à lui attribuer la valeur de vérité « vrai ») 18. Ces possibilités sont récapitulées dans l'organigramme ci-dessous. Nous montrons pourquoi, de notre point de vue, aucune de ces quatre possibilités n'est satisfaisante. 


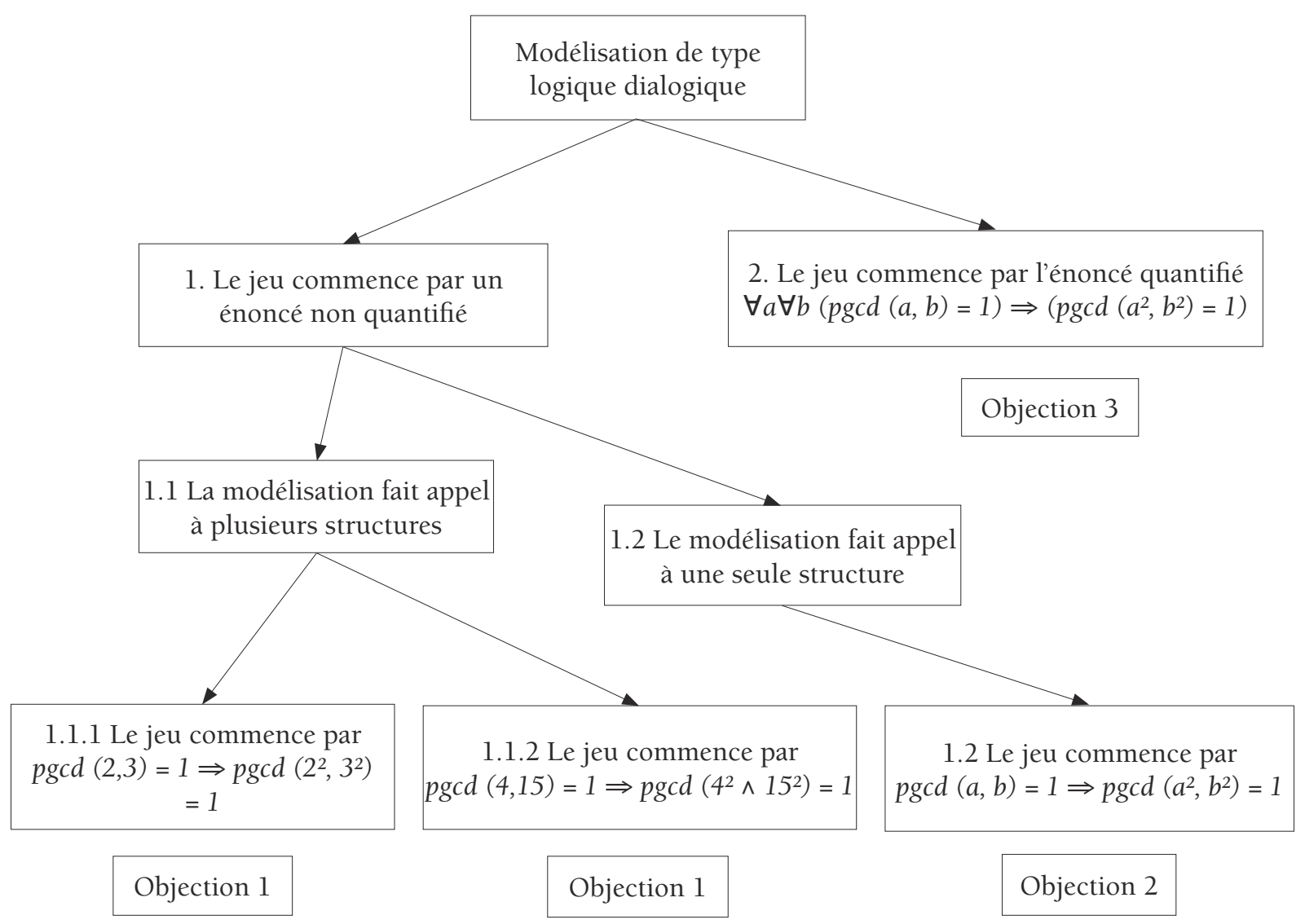

Modélisation 1.1.1 et 1.1.2

Les deux énoncés $\operatorname{pgcd}(2,3)=1 \Rightarrow \operatorname{pgcd}\left(2^{2}, 3^{2}\right)$ $=1$ et $\operatorname{pgcd}(4,15)=1 \Rightarrow \operatorname{pgdc}\left(4^{2}, 15^{2}\right)=1$ induisent chacun un jeu distinct. Dans ces jeux formels, les énoncés atomiques soutenus par le proposant, qui du point de vue sémantique ont été admis comme vrais, sont considérés comme des axiomes (des règles du jeu) afin que le proposant puisse toujours les soutenir. La modélisation 1.1.1 donne donc lieu au tableau suivant:

Axiome:

$\operatorname{pgcd}\left(2^{2}, 3^{2}\right)=1$

\begin{tabular}{|c|c|c|}
\hline & Opposant & Proposant \\
\hline A & & $p g c d(2,3)=1 \Rightarrow p g c d\left(2^{2}, 3^{2}\right)=1$ \\
\hline B & $\operatorname{pgcd}(2,3)=1$ & \\
\hline C & & $\operatorname{pgcd}\left(2^{2}, 3^{2}\right)=1$ \\
\hline
\end{tabular}




\section{Objection 1}

Cette modélisation rompt avec l'unité du dialogue en multipliant les structures de modélisation (il est nécessaire d'utiliser autant de modélisations que de parties du point de vue sémantique). Ensuite, le choix de considérer la proposition $\operatorname{pgcd}\left(2^{2}, 3^{2}\right)$ $=1$ (respectivement $\left.\operatorname{pgcd}\left(4^{2}, 15^{2}\right)=1\right)$ comme un axiome de la modélisation 1.1.1 (respectivement 1.1.2) n'est pas compatible avec la recherche de la fonction dialogique des assertions des élèves. Les « axiomes » font partie des règles structurantes qui contribuent, avec les règles d'usage des connecteurs logiques, à définir la façon dont chacun des jeux peut être joué. Considérer la proposition $\operatorname{pgcd}\left(2^{2}, 3^{2}\right)=1$ comme un axiome revient à accepter que les règles du jeu puissent se définir en cours de partie. Dans ce cas, toute explication en terme de stratégie semble exclue.

\section{Modélisation 1.2}

Le jeu se structure autour de $\operatorname{pgcd}(\mathrm{a}, \mathrm{b})=1 \Rightarrow$ $\operatorname{pgcd}\left(\mathrm{a}^{2}, \mathrm{~b}^{2}\right)=1$ ce qui permet d'unifier la modélisation. Pour les mêmes raisons que précédemment, l'axiome $\operatorname{pgcd}\left(\mathrm{a}^{2}, \mathrm{~b}^{2}\right)=1$ est nécessaire.

Axiome:

$\operatorname{pgcd}\left(\mathrm{a}^{2}, \mathrm{~b}^{2}\right)=1$

\begin{tabular}{|c|c|c|}
\hline & Opposant & Proposant \\
\hline A & & $p g c d(a, b)=1 \Rightarrow p g c d\left(a^{2}, b^{2}\right)=1$ \\
\hline B & $p g c d(a, b)=1$ & \\
\hline C & & $p g c d\left(a^{2}, b^{2}\right)=1$ \\
\hline
\end{tabular}

\section{Objection 2}

Cette modélisation évite la première partie de l'objection 1. Cependant, le nouvel axiome utilisé ne semble pas plus que les axiomes des modélisations 1.1.1 et 1.1.2 pouvoir être considéré comme un règle logique structurant a priori le dialogue. Ensuite le fait que $\mathrm{pgcd}$ $\left(a^{2}, b^{2}\right)=1$ soit une phrase ouverte et contingente (selon les valeurs de $a$ et de $b$ elle est susceptible d'être vraie ou fausse) n'est pas compatible avec le statut d'axiome: il est très peu probable que cette phrase fasse partie des « convictions inébranlables » des élèves.

\section{Modélisation 2}

Axiomes:

$\operatorname{pgcd}\left(2^{2}, 3^{2}\right)=1, \operatorname{pgcd}\left(4^{2}, 15^{2}\right)=1$

\begin{tabular}{|c|c|c|c|c|}
\hline & \multicolumn{2}{|c|}{ Opposant } & \multicolumn{2}{|c|}{ Proposant } \\
\hline A & & & $\forall a \forall b(p \operatorname{gcd}(a, b)$ & $\left(\operatorname{pgcd}\left(a^{2}, b^{2}\right)=1\right)$ \\
\hline B & $a=2 ; b=3$ & $a=4 ; b=15$ & $\operatorname{pgcd}(2,3)=1 \Rightarrow \operatorname{pgcd}\left(2^{2}, 3^{2}\right)=1$ & $\operatorname{pgcd}(4,15)=1 \Rightarrow \operatorname{pgcd}\left(4^{2}, 15^{2}\right)=1$ \\
\hline C & $\operatorname{pgcd}(2,3)=1$ & $\operatorname{pgcd}(4,15)$ & & \\
\hline $\mathrm{D}$ & & & $\operatorname{pgcd}\left(2^{2}, 3^{2}\right)=1$ & $\operatorname{pgcd}\left(4^{2}, 15^{2}\right)=1$ \\
\hline
\end{tabular}

\section{Objection 3}

Les difficultés « chronologiques » liées au statut d'axiome pour les propositions atomiques soutenues par le proposant perdurent. Ensuite, dans le dialogue ci-dessus, l'opposant ne joue pas son rôle: il lui suffirait de faire n'importe quel autre choix en $B$ pour gagner la partie. 


\section{Conclusion}

En rentrant dans le détail de l'analyse, notre objectif était de montrer qu'une modélisation adoptant un point de vue sémantique était plus adaptée pour l'extrait choisi qu'une modélisation construite sur la logique dialogique. Pour traduire un jeu sémantique en un jeu reposant uniquement sur la syntaxe des énoncés il est «naturel » d'essayer de traduire les informations importantes concernant l'interprétation des énoncés atomiques à travers un certain nombre d'axiomes qui joueront le rôle de spécification syntaxique de ces informations. Ceci permet d'obtenir une stratégie reposant seulement sur la syntaxe (Rahman \& Tulenheimo, 2006). Cependant, cette méthode de traduction n'est pas compatible avec notre objectif. La principale difficulté est une difficulté d'ordre chronologique: il ne nous paraît pas pertinent de considérer que les règles du jeu puissent être modifiées en cours de jeu. Pour terminer, on peut remarquer que la correction de Vernant est une autre solution au problème soulevé par Lorenzen lorsqu'il refuse de considérer toutes les propositions comme ayant une valeur de vérité décidable. Ce problème est à la fois un problème fondationnel et un problème pragmatique. Lorenzen avance une réponse fondationnelle en introduisant la notion de proposition d-définies et en considérant des jeux d'intérieur. Vernant propose une solution au problème pragmatique en considérant des jeux d'extérieur dans lesquels les valeurs de vérité des propositions ne sont pas données d'avance. Du point de vue de la modélisation de l'activité mathématique, la correction de Vernant permet d'éviter que le problème chronologique ne s'applique à la modélisation sémantique en permettant aux évaluations des propositions atomiques d'être faites en cours de partie (et non avant de jouer comme dans la version non corrigée).

\section{Le rôle de la sémantique dans les situations de validation}

« Nous pensons avoir montré, que le modèle de Lorenzen, malgré l'intérêt incontestable de l'aspect dialogique qu'il a développé, ne suffit pas pour comprendre et analyser l'activité mathématique. Nous faisons même l'hypothèse qu'il contribue à en donner une image déformée, en exacerbant le travail sur les énoncés au détriment du travail sur les objets, leurs propriétés et les relations mutuelles qu'ils entretiennent. » (DurandGuerrier, 2007, p. 23)

Prolongeant le travail de Durand-Guerrier, nous avons montré qu'il n'est pas souhaitable, au moins dans certains cas, de séparer dans les analyses les objets mathématiques des énoncés les concernant. Au contraire, il semble que pour une partie de l'activité mathématique, le travail sur la forme des énoncés se fasse conjointement avec un travail sur les objets. Comme les autres éléments du milieu, les objets contribuent à la détermination des jeux de langage et à l'émergence des stratégies dans ces jeux. De ce point de vue, il nous semble que la trichotomie de la théorie des situations (situations d'action, situations de formulation et situations de validation) dans laquelle nous avons inscrit ce texte puisse contribuer à masquer l'importance des objets et des actions sur ceux-ci dans la situation de validation. Citons Brousseau à propos du schéma de situation de preuve:

«Les schémas de l'action et de la formulation comportent des processus de correction empirique ou culturelle propres à assurer la pertinence, l'adéquation, l'adaptation ou la conformité des connaissances mobilisées. Mais la modélisation en termes de situation permet de distinguer un nouveau type de formulation. Lémetteur n'y est plus un informateur mais un proposant et le récepteur un opposant. Ils sont supposés posséder les mêmes informations nécessaires. Ils coopèrent dans la recherche de la vérité, c'est-à-dire du moyen de rattacher de façon sûre une connaissance à un champ de savoirs déjà établis, mais s'opposant à chaque instant dès qu'il y a doute. Ils s'occupent ensemble des relations formulées entre un milieu et une connaissance relative à ce milieu. Chacun peut prendre position par rapport à un énoncé, et s'il y a désaccord, demander une démonstration ou exiger que l'autre applique ses déclarations dans la situation d'action avec le milieu. » (Brousseau, 1997, p. 8)

La trichotomie de la théorie des situations n'est donc pas exclusive, en particulier, comme le montre la citation ci-dessus, les actions sur les objets interviennent également dans les situations de validation. L'adéquation de cette description avec la logique dialogique de la véridicité de Vernant (2004) est importante. Cependant, nous considérons que les actions sur les objets n'interviennent pas seulement lorsqu'il s'agit de se mettre d'accord sur un énoncé ${ }^{19}$. Mettre 
des objets (génériques ou individuels) à travers les règles d'instanciation universelle ou existentielle dans le milieu contribue à faire vivre les jeux de validation. De ce point de vue, l'utilisation stratégique des règles du jeu de la validation dépend de ces objets autant qu'elle dépend des autres paramètres de la situation. Autrement dit, la manipulation des objets ne sert pas seulement à confirmer la validité (ou au contraire à l'invalider) d'un énoncé mais aussi à l'émergence des stratégies. Il nous semble que la distinction introduite par Balacheff entre situation de décision et situation de validation permette de préciser ces remarques:

« Dans la situation de décision, les opérations intellectuelles du raisonnement hypothético-déductif (en tant que système légitime et fiable de production d'informations) peuvent être mises en ouvre sans que pour autant une preuve ne soit produite. Les contrôles logiques et sémantiques fonctionnent localement dans le cours de l'élaboration de la solution. Éventuellement, en tant que mathématiciens, nous reconnaîtrons dans ce processus une organisation qui est de l'ordre de la démonstration; mais ici elle est dans le fonctionnement du sujet un outil et non un objet (cf. pour cette distinction Douady, 1985).» (Balacheff, 1987, p. 153)

Selon cette définition, les situations de décision s'opposent à celles de validation dans leur rapport avec la preuve, dans un cas un objectif du dialogue est explicitement la production d'une preuve et dans l'autre l'objectif est celui de la production d'énoncés vrais ${ }^{20}$. Nous pouvons observer dans cette citation que Balacheff partage l'approche de Brousseau à propos du rôle de la sémantique dans ce type de situations puisqu'il lui confère un statut de contrôle des raisonnements plutôt que de construction de ceux-ci. Cependant, les précisions apportées par cette classification permettent de mieux décrire le rôle des actions dans ces situations. Si on réutilise celle-ci pour classer les dialogues, le dialogue concernant la rationalité de $\sqrt{ } 2$ est un dialogue de validation - il a une visée démonstrative (26) et (27) - et le dialogue rapporté sur la conservation de la relation « être premier entre eux » après un passage au carré est un dialogue de décision - l'objectif est d'abord de se mettre d'accord sur la validité de la proposition. Dans le premier, le proposant insiste sur la stratégie qu'il met en œuvre. Les objets n'interviennent pas (nous avons montré qu'une modélisation à partir de la logique dialogique est disponible), les choix d'objets sont des choix formels de lettres. Cette analyse ne constitue pas pour autant un contre-exemple à la thèse selon laquelle le rôle des objets est important dans la construction des stratégies par les élèves. En effet, dans le cas de ce dialogue, la mise en place de la stratégie gagnante du proposant a été prise en charge presque exclusivement par l'enseignante ${ }^{21}$ (les élèves ont d'ailleurs déjà vu une démonstration de l'irrationalité de $\sqrt{2}$ dans leur cours).

Dans le second exemple que nous avons présenté, les objets interviennent d'abord pour contribuer à la décision. Cependant, il nous semble que les élèves, reflétant la culture scolaire (dans laquelle on montre qu'un énoncé est vrai par un travail sur les énoncés et l'on montre qu'un énoncé est faux par un contre exemple) négligent la possibilité que la manipulation des objets, ici les entiers naturels, puisse contribuer à l'émergence d'une preuve (à l'émergence d'une justification pour la stratégie mise en œuvre). Cette possibilité est ignorée au moment où une élève s'engage dans une décomposition de 16 en nombres premiers (20), le groupe préférant s'en remettre au cours. Cette conception de l'activité mathématique comme une activité hypothético-déductive (conçue essentiellement comme l'application du Modus Ponens à partir des théorèmes du cours et des hypothèses de la situation) plutôt qu'une activité expérimentale encourage une sacralisation de l'édifice mathématique et peut, comme dans l'exemple traité, freiner le travail de recherche: les élèves semblent considérer qu'agir sur les objets et chercher une démonstration sont deux activités disjointes ou dit autrement qu'il y a une rupture entre les moments de décisions et les moments de validation. L'activité sémantique semble ici utilisée comme une activité de recherche de contre-exemples, qui lorsqu'elle n'aboutit pas est laissée à l'abandon. 


\section{Conclusions}

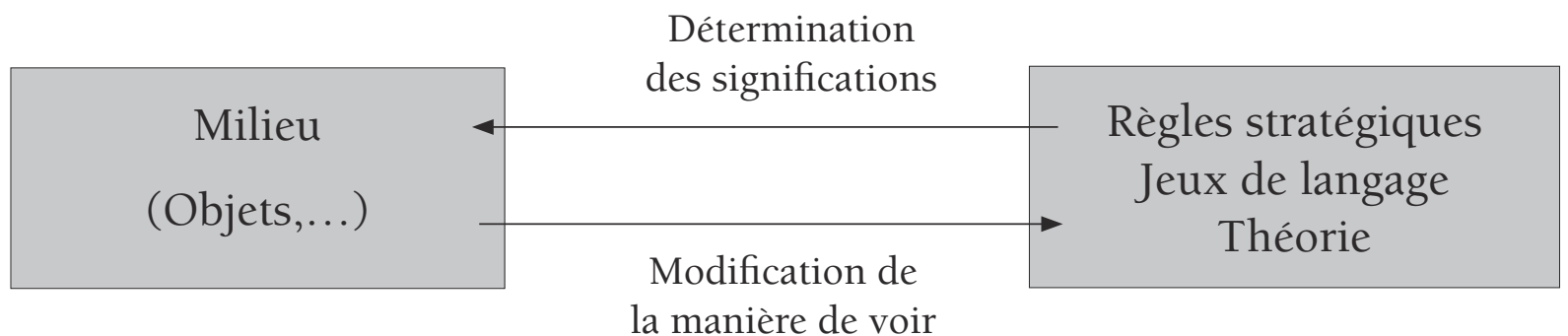

Modélisation des situations de validation

Dans le premier exemple que nous avons traité, un élève expose à un autre élève une preuve. Le dialogue est dans ce cas un dialogue formel, l'élève expose la structure d'un jeu de langage en mettant en évidence des choix stratégiques déjà connus. Il nous semble que la logique dialogique de Lorenzen permet une modélisation de ce type de dialogue que nous avons situé sur le pôle droit de notre schéma. Cependant, dans les situations de validation (au sens large) les objets sont des composants indispensables du milieu et leur manipulation est nécessaire à l'émergence des stratégies. Malgré les citations que nous avons faites de Brousseau et de Balacheff concernant la place des objets dans les situations de validation (ces citations insistent plus sur la flèche du bas du schéma que sur celle du haut), cette position s'accorde avec les principes de la théorie des situations tant celle-ci insiste sur l'importance des situations pour montrer (pôle gauche de notre schéma) ce qui ne peut être dit (pôle droit du schéma):

"Cette position commune à Brousseau et Wittgenstein marque la dimension anthropologique de la manière dont l'un et l'autre examinent les conditions susceptibles de montrer ce qui ne peut être dit, et de décrire les conditions de la genèse d'une pratique par et dans laquelle les mots, les concepts... les savoirs prennent leurs sens. » (Sarrazy, 2005, p. 378)

En intégrant les objets, la sémantique GTS de Hintikka permet des modélisations des activités de recherche qui tiennent compte conjointement des deux pôles. Si l'on considère que l'émergence de stratégies dans une situation est rendue possible par des allers-retours entre les deux pôles du schéma à travers des corrections de la théorie - le plus souvent implicite - (flèche du haut) et des confrontations avec les objets (flèche du bas) (Dias et DurandGuerrier, 2005, Lakatos, 1984) une modélisation de ce type paraît nécessaire. Il s'agit donc de mettre en avant le rôle des objets mathématiques dans la relation dialectique outil/objet concernant la preuve. Bien qu'il n'y ait pas de rupture franche, nous avons montré que les dialogues pouvaient être de nature différente dans les situations de décision et dans les situations de validation dans le sens où nous avons dû les modéliser de manières différentes (non traductibles d'un point de vue à l'autre). Cette distinction entre décision et validation est vraisemblablement en partie parallèle à celle établie par Marion (2006a) entre les jeux de recherche et de résolution ( "games of seeking and finding ») et les jeux d'assertions ( " games of giving and asking for reasons »), les premiers servant de support à la sémantique selon la théorie des jeux de Hintikka et les seconds étant des jeux d'intérieur (la logique dialogique de Lorenzen en est une formalisation). En effet, dans un jeu de recherche et de résolution la question en jeu est celle de la vérité d'une proposition alors qu'à travers les jeux d'assertions l'accent est mis sur la justification des propositions. Selon Marion (2006a, p. 261), chacun de ces deux types de jeux peut être vu comme une extension raisonnable des idées Wittgensteiniennes puisque les jeux de recherche et de résolution et les jeux d'assertions (sans regarder davantage à leur formalisation) ne peuvent pas être exclus a priori de ce que Wittgenstein entend par «jeux de langage». Du point de vue de la modélisation, ce qui distingue 
ces jeux nous semble être l'intervention d'éléments extra-dialogiques et en particulier des objets d'une structure d'interprétation. Nous avons essayé de montrer que faire une hypothèse réaliste, assumant l'existence d'une structure d'interprétation remplie d'objets mathématiques, est un élément important de l'analyse des dialogues de recherche et de résolution. En particulier, l'explication de la possibilité d'une recherche qui ne sache pas déjà ce qu'elle va trouver passe par le dépassement de ce que nous avons appelé le problème chronologique: il s'agit de réussir à expliquer comment il est possible d'acquérir de nouvelles connaissances sans que celles-ci ne soient déjà contenues dans les règles d'usage du jeu de la recherche.

Notre principal objectif dans cet article a été de montrer qu'une modélisation s'appuyant sur la seule syntaxe (le pôle droit du schéma) n'y parvient pas. Une telle modélisation ne peut pas rendre compte des interactions avec le milieu au cours du temps. Il nous a alors paru indispensable d'intégrer une dimension sémantique (le pôle gauche du schéma) et une dimension transactionnelle qui prenne en charge les interactions avec le milieu (les deux flèches entre les pôles du schéma). 


\section{NOTES}

1. Pour « Game Theoretical Semantics» (Hintikka \& Sandu, 1997).

2. Nous dirons ensuite « théorie des situations » (Brousseau, 1998).

3. Cette correction est décrite dans (Vernant, 2004) ou pour une version anglaise dans (Vernant, 2007).

4. «Et l'usage qu'il fera du mot que je lui explique montre la façon dont il comprend l'explication » (Wittgenstein, 2004, § 29))

5. «L'expression « jeu de langage» doit ici faire ressortir que parler un langage fait partie d'une activité, ou d'une forme de vie» (Wittgenstein, 2004, § 23). Sarrazy (2005) propose une comparaison plus complète de ces auteurs.

6. «Une signification d'un mot est un mode de son utilisation. En effet cette signification est ce que nous apprenons au moment où le mot est incorporé dans notre langage. » (Wittgenstein, 1976, § 61)

"C'est pourquoi il y a une correspondance entre les concepts de « signification » et de " règle ». (Wittgenstein, $1976, \S 62)$

7. Nous discuterons cette trichotomie dans la quatrième partie de ce texte.

8. Mathé \& Barrier (à paraître) en propose une analyse didactique dans le cadre de la construction du vocabulaire géométrique au cycle 3 de l'école primaire en France.

9. Nous marquons par une étoile* le premier usage d'un terme qui est défini en annexe.

10. La question de savoir si il existe des nombres parfaits impairs est ouverte.

11. Ce joueur peut tout aussi bien être le proposant que l'opposant. Par exemple, le rôle d'attaquant et de défenseur est inversé à chaque usage de la règle concernant la négation.

12. La thèse (Battie, 2003) est disponible sur internet à l'adresse suivante:

http://tel.archives-ouvertes.fr/docs/00/14/10/80/PDF/BATTIE_These. pdf

13. Le vocabulaire d'usage en didactique concernant les propositions que nous appelons « axiomes» serait certainement celui de "modèle implicite d'action » ou de "théorème en acte ». Nous préférons le terme d'« axiome » puisque localement ces propositions structurent la démarche langagière (de manière plus ou moins implicite) et ne sont, à l'intérieur de celle-ci, pas déductibles d'autres propositions.

14. Lorenz distingue pour chaque action verbale « le point de vue du Je » et « le point de vue du Tu » (Lorenz, 2003, p. 59).

15. Barrier (à paraître) montre à travers l'analyse d'une preuve de Bolzano qu'il est parfois difficile de faire un choix entre des interprétations divergentes (faisant appel à des règles différentes) d'un même corpus.

16. C'est la raison pour laquelle nous avons préféré une modélisation faisant appel à une axiomatique pour la première partie du dialogue plutôt qu'une modélisation qui considérerait les propositions qui font office d'axiomes dans notre présentation comme des assertions préliminaires de l'opposant.

17. Ce théorème est valide. Une preuve est proposée dans l'annexe mathématique.

18. Cette restriction, favorable au type de modélisation critiqué, nous permet de condenser l'analyse.

19. Durand-Guerrier $(2007$, p. 1) fait «l'hypothèse d'une centration excessive sur le rôle du contre-exemple dans l'activité de preuve en mathématiques ».

20. Hintikka (2007) aborde cette distinction p. 60.

21. Voir l'annexe III. 


\section{BIBLIOGRAPHIE}

Balacheff, N. (1987). Processus de preuve et de validation. Educational Studies in Mathematics, 18/2, 147-176.

Barrier, T. (à paraître). Quantification et Variation en Mathématiques: perspectives didactiques issues de la lecture d'un texte de Bolzano. Proceedings of the 5 th International Colloquium on the Didactics of Mathematics, Rethymnon, Crete, 17-19 April 2008.

Battie, V. (2003). Spécificités et potentialités de l'arithmétique pour l'apprentissage du raisonnement mathématiques. Thèse, Université Paris 7, Paris.

Battie, V. (2007). Exploitation d'un outil épistémologique pour l'analyse de raisonnements d'élèves confrontés à la résolution de problèmes en arithmétique. Recherches en didactique des mathématiques, 27, 9-44.

Brousseau, G. (1997). La théorie des situations didactiques. Cours donné lors de l'attribution à Guy Brousseau du titre de Docteur Honoris Causa de l'Université de Montréal, Montréal, Canada, Juin.

Brousseau, G. (1998). Théorie des situations didactiques. Grenoble: La Pensée Sauvage.

Dias, T. \& Durand-Guerrier, V. (2005). Expérimenter pour apprendre en mathématiques. Repères IREM, 60, 61-78.

Douady, R. (1985). The interplay between different settings. Tool-Object dialectic in the extension of mathematical ability. Proceedings of the Ninth International Conference for the Psychology of Mathematics Education, II, 33-52

Durand-Guerrier, V. (2007). Retour sur le schéma de la validation explicite dans la théorie des situations didactiques, à la lumière de la théorie des modèles de Tarski. Actes du colloque Didactiques: quelles références épistémologiques?, Bordeaux, France, mai 2005.

Durand-Guerrier, V., Héraud, J.-L., \& Tisseron, C. (Eds.). (2006). Jeux et Enjeux de Langage dans l'Elaboration des Savoirs en Classe. Lyon: Presse Universitaire de Lyon.

Guernier, M.-C., Durand-Guerrier, V., \& Sautot, J.-P. (Eds.). (2007). Interactions Verbales, Didactiques et Apprentissages, Besançon: Presse Universitaire de Franche-Comté.

Hintikka, J. (1994). Fondements d'une théorie du langage. Paris: Presses Universitaires de France.

Hintikka, J. (1996). La philosophie des mathématiques chez Kant. Paris: Presses Universitaires de France.

Hintikka, J. (2007). Les Principes des Mathématiques Revisités (M. Rebuschi, Trad.). Paris: Vrin. (Édition originale, 1996).

Hintikka, J., \& Kulas, J. (1983). The game of language. Studies in Game-Theoretical Semantics and its Application. Dordrecht, Boston, Lancaster: Reidel.

Hintikka, J. \& Sandu, G. (1997). Game-Theoretical Semantics. In J. Van Benthem \& A. Ter Meulen (Eds.), Handbook of Logic and Language (p.. 361-410). Amsterdam: Elsevier.

Lakatos, I. (1984). Preuves et Réfutations (N. Balacheff \& J. M. Laborde, Trad.). Paris : Hermann. (Édition originale 1976).

Lorenz, K. (2001). Basic Objectives of Dialogue Logic in Historical Perspective. Synthese, 127, 255-263.

Lorenz, K. (2003). Le dialogue comme sujet et méthode de la philosophie. In F. Armengaud, M.-D. Popelard $\&$ D. Vernant (Eds.), Du Dialogue au Texte: Autour de Francis Jacques (p.. 49-61). Paris: Kimé.

Lorenz, K. (2006). Logic as a Tool of Science versus Logic as a Scientific Subject. In J. Van Benthem, G. Heinzmann, M. Rebuschi \& H. Visser (Eds.), The Age of Alternative Logics: Assessing Philosophy of Logic and Mathematics Today (p.. 299-310). Dordrecht/Boston/London: Springer.

Lorenzen, P. (1967). Métamathématiques. Paris: Gauthier-Villars.

Marion, M. (2006a). Hintikka on Wittgenstein: From Language-Games to Game Semantics. In T. Aho \& A.-V. Pietarinen (Eds.), Truth and Games: Essays in honour of Gabriel Sandu, Acta Philosophica Fennica, 78 (p.. 255-74). 
Marion, M. (2006b). Why Play Logical Games. In O. Majer, A.-V. Pietarinen \& T. Tulenheimo (Eds.), Logic and Games, Foundational Perspectives. Dordrecht: Springer.

Mathé, A.-C., \& Barrier, T. (à paraître). Jeux et enjeux de langage dans la construction d'un vocabulaire spécifique et de références partagées en géométrie en cycle 3. Atelier de la XIVème Ecole d'été de Didactique des Mathématiques. Sainte-Livrade, France, Aout 2007.

Rahman, S. \& Tulenheimo, T. (2006). From Games to Dialogues and Back. In O. Majer, A.-V. Pietarinen \& T. Tulenheimo (Eds.), Logic and Games, Foudational Perspectives. Dordrecht: Springer.

Sarrazy, B. (2005). La théorie des situations: une théorie anthropologique des mathématiques? In P. Clanché, M.-H. Salin \& B. Sarrazy (ed.) Sur la théorie des situations. Question, Réponse, Ouverture. Hommage à Guy Brousseau (p.. 375-390). Grenoble: La pensée sauvage.

Vernant, D. (2004). Pour une logique dialogique de la véridicité. Cahier de linguistique française, 26, 87-111.

Vernant, D. (2005). The Limits of a Logical Treatment of Assertion. In D. Vanderveken (Ed.), Logic Thought and Action (p.. 267-288). Dordrecht: Springer.

Vernant, D. (2007). The dialogical Logic of veridicity. In A. Trognon (Ed.), Logic and Dialogue. Presse Universitaires de Nancy.

Wittgenstein, L. (1976). De la Certitude (J. Fauve, Trad.). Paris: Gallimard. (Édition originale, 1969).

Wittgenstein, L. (2004). Recherches philosophiques (F. Dastur, M. Elie, J.-Gautero, D. Janicaud \& E. Rigal, Trad.). Paris: Gallimard. (Édition originale, 1953) 


\section{ANNEXE I: ANNEXE MATHÉMATIQUE}

Dans cette annexe nous précisons à l'intention du lecteur non spécialiste de mathématiques les définitions et résultats employés dans l'article.

Divisibilité. Au lycée, ce concept concerne les nombres entiers. Pour deux entiers $a$ et $b$ donnés, on dit que $a$ divise $b$ (que $b$ est un multiple de $a$, ou encore que $a$ est un diviseur de $b$ ) si il y a un certain entier $k$ pour lequel on peut écrire $b=k a$. Par exemple, 1,4 ou 7 sont des diviseurs de 28 tandis que 3 n'est pas un diviseur de 28.

Nombre parfait. Un nombre parfait est un entier naturel plus grand que 2 dont la somme des diviseurs autres que lui même lui est égal. Par exemple $6=1+2+3$ et $28=1+2+4+7+14$ sont des nombres parfaits alors que 10 ne l'est pas $(1+2+5=8)$. Pour le moment, tous les nombres parfaits connus sont pairs.

Plus Grand Commun Diviseur. Le pgcd de deux entiers est le plus grand diviseur qui soit commun aux deux entiers. Par exemple, pgcd $(6,28)=2, \operatorname{pgcd}(21,28)=7, \operatorname{pgcd}(4,15)=1$.

Nombres premiers entre eux. Deux entiers sont premiers entre eux lorsqu'ils n'ont pas de diviseurs en commun autres que 1 . Autrement dit, deux entiers sont premiers entre eux si leur pgcd vaut 1 . Par exemple, 4 et 15 sont premiers entre eux ainsi que 28 et 15 tandis que 12 et 21 ne le sont pas (ils sont tous deux divisibles par 3).

Nombre rationnel. Un nombre réel est dit rationnel lorsqu'il est possible de l'écrire sous la forme d'une fraction de nombres entiers. Un nombre est dit irrationnel si il n'est pas rationnel. Par exemple, 0,62 $=\underset{100}{\operatorname{es}(\mathrm{tn}}$ nombre rationnel.

Fraction irréductible. Une fraction est dite irréductible lorsque son numérateur et son dénominateur n'ont pas de diviseur commun par lequel simplifier la fraction. Autrement dit, une fraction est dite irréductible si son numérateur et son dénominateur sont premiers entre eux. Par exemple, $\frac{162 e s t}{100}$ pas irréductible parce que 62 et 100 sont divisibles par 2. Par contre, est une $\frac{\text { Praction irréductible puisque } p \text { gcd }}{50}(31,50)=1$.

Nombre premier. Un nombre entier est dit premier si il n'a pas d'autres diviseurs que 1 et lui-même. Par exemple, 7 est un nombre premier, 19 ou 79 aussi mais pas 39 qui divisible par 3.

Théorème de Gauss. Lorsqu'un nombre entier $a$ divise le produit $b c$ des nombres entiers $b$ et $c$ et lorsque les nombre $a$ et $b$ sont premiers entre eux alors $a$ divise $c$.

Preuve de $(p \operatorname{gcd}(a, b)=1) \Rightarrow\left(\operatorname{pgcd}\left(a^{2}, b^{2}\right)=1\right)$. Nous utilisons le résultat selon lequel tout entier naturel admet une décomposition unique (à l'ordre des termes près) en produit facteurs premiers (par exemple $28=2$ $\times 2 \times 7$ ou $30=2 \times 3 \times 5)$ :

Cette écriture donne pour $a$ et $b: a=p_{1} p_{2} \ldots p_{n-1} p_{n}$ et $b=q_{1} q_{2} \ldots q_{m-1} q_{m}$

On obtient alors les écritures en produit de facteurs premiers pour $a^{2}$ et $b^{2}$ :

$\mathrm{a}^{2}=p_{1} p_{1} p_{2} p_{2} \ldots p_{n-1} p_{n-1} p_{n} p_{n}$ et $b^{2}=q_{1} q_{1} q_{2} q_{2} \ldots q_{m-1} q_{m-1} q_{m} q_{m}$

Dire que $\operatorname{pgcd}(a, b)=1$, c'est dire que les décompositions de $a$ et de $b$ n'ont aucun facteur en commun. Lorsque c'est le cas, les décompositions de $a^{2}$ et $b^{2}$ n'en n'ont pas non plus. 


\section{ANNEXE II : ANNEXE LOGIQUE}

Constantes logiques. Ce sont les éléments avec lesquels on construit les formules. Par exemple si p et q sont des formules alors $\mathrm{p} \wedge \mathrm{q}, \exists \chi \rho, \ldots$ sont des formules. Nous utilisons ici 6 constantes logiques: la conjonction $\wedge$ (pour formaliser « et »), la disjonction $\vee$ (pour formaliser « ou »), le quantificateur universel $\forall$ (pour formaliser « pour tout »), le quantificateur existentiel $\exists$ (pour formaliser « il existe »), la négation $\neg$ (pour formaliser «non ») et le conditionnel $\Rightarrow$ (pour formaliser « implique »).

Constantes extra-logiques. Ce sont les éléments des propositions autres que les variables et les constantes logiques. Autrement dit, pour ce qui nous intéresse ici, les symboles de constantes, de fonctions et de prédicats (et de relations).

Par exemple, dans la proposition a $\vee \neg$ a, il n'y a que la constante extra-logique $a$. Dans la proposition $\forall a \forall b(p \operatorname{gcd}(a, b)=1) \Rightarrow\left(p g c d\left(a^{2}, b^{2}\right)=1\right)$, les constantes extra-logiques sont la relation d'égalité, la fonction notée ${ }^{2}$ et la fonction $p g c d$.

Propositions atomiques. Ce sont les propositions dans lesquelles il n'y a pas de constante logique. Par opposition, une proposition est dite complexe si elle est construite avec des constantes logiques. Par exemple, la proposition $a=2 q$ est une proposition atomique alors que $\mathrm{a}=2 \mathrm{q} \wedge \mathrm{b}=2 \mathrm{q}$ 'ou $\exists \mathrm{q}, \mathrm{a}=2 \mathrm{q}$ sont des propositions complexes. 


\section{ANNEXE III : EXTRAIT DES ÉPISODES 4 ET 5}

Les interventions de l'enseignante sont en italiques et précédées d'un $P$.

\begin{tabular}{|c|c|}
\hline Episode 4 & Episode 5 \\
\hline$P:$ Bon, vous vous en sortez? & P: Alors, est-ce que les idées de Al aboutissent? \\
\hline Non. & Non. \\
\hline P: Pas du tout?! & P: Non?! \\
\hline Non. & $\underline{\text { inaudible }}$ \\
\hline-- & P: Mais si elle en avait tout à l'heure. \\
\hline 4. $\quad P$ : Attends, racine de 2 diviseur ça veut rien dire parce que & Al : $\mathrm{a}^{2}$ est multiple de 2 et pis euh/ \\
\hline racine de 2 c'est pas un entier il faut forcément que tu travailles avec & $P:$ et a alors, a il est quoi? \\
\hline un entier. Faut forcément travailler avec ça. Voilà, c'est là dessus qu’il & $\mathrm{a}^{2}$ est multiple de 2 . \\
\hline faut travailler! & $P: a^{2}$ est multiple de 2 \\
\hline 5. Al : C'était une bonne idée de mettre au carré? & Al : Ben il est multiple de 2 (hésitant). \\
\hline $\begin{array}{l}\text { 6. P: Oui bien-sûr. C'était, C'était forcément une bonne idée } \\
\text { puisque... si tu veux pouvoir travailler sur les entiers, faut, faut avoir }\end{array}$ & $\begin{array}{l}\text { 29. P: Forcément ou pas? Oui ou non je } \\
\text { sais pas j'te demande! }\end{array}$ \\
\hline ça. $\quad$ Ben/ & $\begin{array}{l}30 \text {. Ca fait a fois a multiple de } 2 \text {, donc le carré est } \\
\text { multiple de } 2 \text {. }\end{array}$ \\
\hline P: Donc c'est là dessus qu'il faut travailler. & 31. $\quad$ Oui. \\
\hline P: Ben continue. Qu'est-ce que tu peux en déduire? & 32. P: Tu essaies de m'écrire ça et puis si a est multiple de \\
\hline 2 divise $a^{2}$ & a tu vas peut-être pouvoir l'écrire! Comment tu l'écrirais que a est \\
\hline P: Donc qu'est ce que tu peux en déduire sur a? Par & multiple de 2 ? \\
\hline$e_{-1}$ & 33. $a$ égal $2 k$. \\
\hline Qu'il est supérieur à inaudible. & P: Voilà! \\
\hline P: Tu peux en déduire un peu plus que ça. & inaudible \\
\hline C'est un multiple de 2 . & P: Attends, avant de dire je vais être bloquée, essaye! \\
\hline $\begin{array}{l}\text { 15. P: Voilà! Ben continue dans cette voie. Suis ta, suis les } \\
\text { idées de Al elles m’ont l'air bonnes. }\end{array}$ & $\begin{array}{l}37 . \quad \text { Al: Ah oui, ben forcément, oui ben oui! } \\
\text { a est multiple de } 2 \text {. }\end{array}$ \\
\hline $\begin{array}{l}16 . \quad \text { Eh oui évidemment. } \\
\text { (Rires) }\end{array}$ & $\begin{array}{l}38 . \\
\text { (rires) }\end{array}$ \\
\hline J'l'ai toujours dit. Euh... & $\begin{array}{l}\text { 39. P: Bon ben alors du coup tu vas pouvoir écrire. Ecris-le } \\
\text { que a est multiple de } 2 \text { ! Qu'est-ce que ça te donne?! }\end{array}$ \\
\hline Dis-nous Al tout ce qui te passe par la tête. & $\begin{array}{ll}40 . & \text { Al : a égal } 2 q \\
41 . & P: \text { Voilà. } \\
42 . & \text { Al : Et donc quand... } \\
\text { 43. } & \text { P: Reprends le moral A2. } \\
\text { (rires) } & \\
44 . & \text { J'attends. } \\
45 . & \text { P: Ah non! Tu n'attends pas. Tu aides A1. } \\
\\
\text { P s'éloigne... }\end{array}$ \\
\hline
\end{tabular}

